\title{
Downregulation of BZW2 inhibits osteosarcoma cell growth by inactivating the Akt/mTOR signaling pathway
}

\author{
DONG-DONG CHENG ${ }^{*}$, SHI-JIE LI* , BIN ZHU, TING YUAN, QING-CHENG YANG and CUN-YI FAN \\ Department of Orthopedics, Shanghai Jiaotong University Affiliated Sixth People's Hospital, Shanghai 200233, P.R. China
}

Received January 15, 2017; Accepted July 4, 2017

DOI: $10.3892 /$ or.2017.5890

\begin{abstract}
Osteosarcoma is the most common malignant bone tumor in adolescents. The function of basic leucine zipper and W2 domains 2 (BZW2) in tumor progression has been reported. However, the role and mechanisms of BZW2 in osteosarcoma remain to be determined. The aim of the present study was to reveal the expression and biological functions of BZW2 in osteosarcoma and to elucidate the proximal mechanisms underlying these functions. The expression of BZW2 in osteosarcoma tissues and cell lines was assessed by qRT-PCR, western blotting and immunohistochemistry. BZW2 overexpression was detected in osteosarcoma cell lines. Clinically, BZW2 expression was higher in osteosarcoma tissues than in corresponding non-tumor tissues and was associated with advanced Enneking stage and tumor recurrence. The knockdown of BZW2 using siRNA inhibited osteosarcoma cell proliferation, colony-forming ability, and the cell cycle at the G2/M phase in vitro. Host signaling pathways affected by BZW2 were detected using a PathScan Intracellular Signaling Antibody Array kit. These data demonstrated that the knockdown of BZW2 suppresses protein phosphorylation in the Akt/mTOR signaling pathway. These observations suggest that BZW2 is upregulated and has a pro-tumor effect in osteosarcoma via activation of the Akt/mTOR signaling pathway and thus is a potential target for gene therapy.
\end{abstract}

\section{Introduction}

Osteosarcoma, the most common primary malignancy of bones, is histologically characterized by the presence of

Correspondence to: Dr Cun-Yi Fan or Dr Qing-Cheng Yang, Department of Orthopedics, Shanghai Jiaotong University Affiliated Sixth People's Hospital, 600 Yishan Road, Shanghai 200233, P.R. China

E-mail: fancunyi888@hotmail.com

E-mail: tjyqc@163.com

*Contributed equally

Key words: BZW2, Akt/mTOR signaling pathway, proliferation, osteosarcoma malignant mesenchymal cells with a high rate of pulmonary metastasis $(1,2)$. In the past, the 5-year survival rate for osteosarcoma was $\sim 10-20 \%$, indicating a very poor prognosis (3). Multidisciplinary treatment, including limb salvage surgery and multidrug neoadjuvant chemotherapy, has improved the cumulative 5-year survival to $60-80 \%$ (4). Thus, osteosarcoma is no longer incurable. However, the overall survival has remained nearly unchanged in the last three decades owing to genetic, epigenetic and biological complexities (5). Therefore, elucidating the molecular mechanisms underlying osteosarcoma is critical for developing new therapeutic strategies to improve the prognosis of patients with osteosarcoma.

Basic leucine zipper and W2 domains 2 (BZW2) is a member of the bZIP superfamily of transcription factors. It is involved in cell-cell adhesion via cadherin binding. Guo et al reported that BZW2 is a novel E-cadherin-interacting protein based on a quantitative proteomic analysis (6). BZW1, another member of the bZIP superfamily, is a novel proliferation regulator in salivary mucoepidermoid carcinoma. The knockdown of BZW1 significantly inhibited the proliferation and metastasis of Mc3 cells and a high expression of BZW1 has been detected in high-grade mucoepidermoid carcinoma (7). However, for BZW2, the potential roles and underlying mechanisms in tumorigenesis remain elusive.

Accumulating studies have shown the importance of the $\mathrm{PI} 3 \mathrm{~K} / \mathrm{AKT} / \mathrm{mTOR}$ signaling pathway in tumorigenesis $(8,9)$. Sun et al reported that the activation of the PI3K/AKT/mTOR signaling pathway is correlated with tumor progression and decreases patient survival for those with urothelial carcinoma of the urinary bladder (10). Similarly, overexpression of the $\mathrm{PI} 3 \mathrm{~K} / \mathrm{Akt} / \mathrm{mTOR}$ pathway proteins in sialyl-Tn antigen-positive muscle invasive bladder cancer is independently associated with a high risk of cancer-related deaths (11). However, it is not clear whether the Akt/mTOR signaling pathways are involved in the BZW2-induced effects on osteosarcoma.

In the present study, we investigated the expression of BZW2 in human osteosarcoma cell lines and an osteoblast cell line. BZW2 was overexpressed in osteosarcoma cell lines and osteosarcoma tissues. The functions of BZW2 were further examined in osteosarcoma cells. Our results demonstrated that the knockdown of BZW2 hinders osteosarcoma cell proliferation and colony-formation ability by inhibiting the G2/M cell cycle transition. The mechanism underlying the function of BZW2 was also investigated. Decreased BZW2 downregulated the Akt/mTOR signaling pathway in osteosarcoma 
cells. Notably, we found that BZW2 expression was positively correlated with the Enneking stage and the recurrence of osteosarcoma. To the best of our knowledge, this is the first evidence that the overexpression of BZW2 is a prerequisite for the development and progression in human osteosarcoma.

\section{Materials and methods}

Cell lines and cell culture. Two osteosarcoma cell lines (MNNG/HOS and U2OS) and one human osteoblast cell line (hFOB 1.19) were used in the present study. All three cell lines were obtained from the Cell Bank of the Chinese Academy of Sciences (Shanghai, China). They were cultured in either Dulbecco's modified Eagle's medium (MNNG/HOS) or RPMI-1640 medium (U2OS) containing 10\% fetal bovine serum (Bio-west, Logan, UT, USA), $100 \mathrm{U} / \mathrm{ml}$ penicillin, and $100 \mathrm{mg} / \mathrm{ml}$ streptomycin (both from Sigma-Aldrich, St. Louis, MO, USA). The cells were maintained at $37^{\circ} \mathrm{C}$ in a humidified atmosphere containing $5 \% \mathrm{CO}_{2}$. The hFOB 1.19 cell line was cultured according to the established American Type Culture Collection (ATCC; Mannasas, VA, USA) protocols.

Human osteosarcoma samples. From 2015 to 2016, 50 patients with osteosarcoma were treated at Shanghai Jiaotong University Affiliated Sixth People's Hospital. They received primary surgical treatment and preoperative and postoperative neoadjuvant therapy. Each osteosarcoma sample along with a corresponding adjacent non-tumor tissue sample was obtained during surgery. The samples were immediately frozen in liquid nitrogen after resection and stored at $-80^{\circ} \mathrm{C}$ in a refrigerator. Ethics approval was obtained from the local hospital Ethics Committees, and written informed consent was obtained from each patient prior to sample collection (YS-2016-064, 24 February 2016).

RNA extraction and $q R T-P C R$ analysis. Total RNAs of human tissue samples and cultured cells were purified using TRIzol reagent (Invitrogen, Carlsbad, CA, USA). cDNA was synthesized using a PrimeScript RT Reagent kit (Takara, Shiga, Japan). qRT-PCR was performed using SYBR-Green Premix Ex Taq (Takara) on an ABI 7500 PCR system (Applied Biosystems, Foster City, CA). All reactions were performed in $10-\mu 1$ reaction volumes in triplicate. The primer sequences were as follows: BZW2 forward, 5'-GGAGCTTTC CGACTTCCTCC-3' and reverse, 5'-AAGCACCACCTCCTT GATCG-3'; $\beta$-actin forward, 5'-TTGTTACAGGAAGTCC CTTGCC-3' and reverse, 5'-ATGCTATCACCTCCCCTGT GTG-3'.

Protein extraction and western blot analysis. Lysates were extracted from cultured cells using T-PER Protein Extraction Reagent (Thermo Fisher Scientific, Inc., Waltham, MA, USA) containing PhosSTOP (Roche, Basel, Switzerland) and Complete Mini.Equal amounts of proteins were electrophoresed and transferred to nitrocellulose membranes or polyvinylidene fluoride membranes (Millipore, Billerica, MA, USA). After they were blocked in 5\% non-fat milk, the membranes were incubated with the following primary antibodies: BZW2 (polyclonal; 1:500; \#21001-1-AP; Proteintech, Wuhan, China), mTOR (total) (monoclonal; 1:1,000; \#2983), mTOR (Ser2448) (monoclonal; 1:1,000; \#5536), Akt (total) (monoclonal; 1:1,000; \#4691), Akt (Thr308) (monoclonal; 1:1,000; \#13038) (all from Cell Signaling Technology), or $\beta$-actin (polyclonal; 1:5,000; \#20536-1-AP; Proteintech). The secondary antibody was goat anti-rabbit IgG (1:5,000; \#A0545; Sigma-Aldrich). Visualization was performed using SuperSignal West Femto Maximum Sensitivity Substrate (Thermo Fisher Scientific, Inc.).

siRNA transfection. Human BZW2 siRNA and non-specific control (NC) siRNA were synthesized by RiboBio (Guangzhou, China) and were transfected into cells using Lipofectamine 2000 reagent (Invitrogen) following the manufacturer's protocol. The sequence of the siRNA targeting $B Z W 2$ was GGTCTTCTGTGGACATGTA. For proliferation and cell cycle analyses as well as RNA extraction and western blotting, cells were used $48 \mathrm{~h}$ after transfection.

Cell proliferation and colony formation assays. For the cell proliferation assay, $48 \mathrm{~h}$ after transfection, the cells were seeded into a 96-well plate (3,000 cells/well). A 10- $\mu$ l aliquot of Cell Counting Kit-8 (CCK-8) solution (Dojindo, Kumamoto, Japan) was added to triplicate wells and incubated for $2 \mathrm{~h}$. The absorbance at $450 \mathrm{~nm}$ was assessed. Each measurement was performed in triplicate, and experiments were repeated twice. For the colony formation assay, $48 \mathrm{~h}$ after siRNA transfection, MNNG/HOS or U2OS cells $\left(1 \times 10^{3}\right.$ cells/well) were cultured in 6-well plates for 10 days, and then subjected to $100 \%$ methanol fixation for $30 \mathrm{~min}$ and $0.1 \%$ crystal violet staining for $30 \mathrm{~min}$. The cell colonies were counted and all assays were independently performed in triplicate.

Cell cycle analysis. Forty-eight hours after transfection, cells were collected and fixed with $70 \%$ ethanol. The cells were then stained with $50 \mu \mathrm{g} / \mathrm{ml}$ of propidium iodide containing RNase I (both from Kaiji Biological Inc., Nanjing, China), followed by analysis using a FACSCalibur flow cytometer (BD Biosciences, San Jose, CA, USA). The results were analyzed using ModFit software (BD Biosciences). Assays were independently performed three times.

Intracellular signaling array. Forty-eight hours after transfection, MNNG/HOS and U2OS cells were harvested and lysed on ice with $0.1 \mathrm{ml}$ of cell lysis buffer containing a cocktail of protease inhibitors for $5 \mathrm{~min}$. The lysates were centrifuged at $10,000 \mathrm{x} \mathrm{g}$ at $4^{\circ} \mathrm{C}$ for $10 \mathrm{~min}$. Intracellular signaling molecules were detected using a PathScan ${ }^{\circledR}$ Intracellular Signaling Array kit (\#7744; Cell Signaling Technology) according to the manufacturer's procedure. Fluorescent images of the slides were captured using the Odyssey ${ }^{\circledR}$ Infrared Imaging System (LI-COR, Lincoln, NE, USA) and the intensities of the spots were quantified using Image Studio analysis software.

Immunohistochemistry. A standard immunohistochemistry (IHC) staining procedure was followed. Briefly, paraffin-embedded sections were cut at $4 \mu \mathrm{m}$, dewaxed in xylene, and heated in a microwave at $60^{\circ} \mathrm{C}$ for $20 \mathrm{~min}$ in EDTA buffer ( $\mathrm{pH}$ 9.0) for antigen retrieval. For each slide, endogenous peroxidase activity was blocked by $10 \mathrm{~min}$ of incubation in $0.3 \%$ $\mathrm{H}_{2} \mathrm{O}_{2}$, followed by incubation at $37^{\circ} \mathrm{C}$ with a $1: 100$ dilution of the primary antibody against BZW2 (Proteintech) or p-AKT 
A

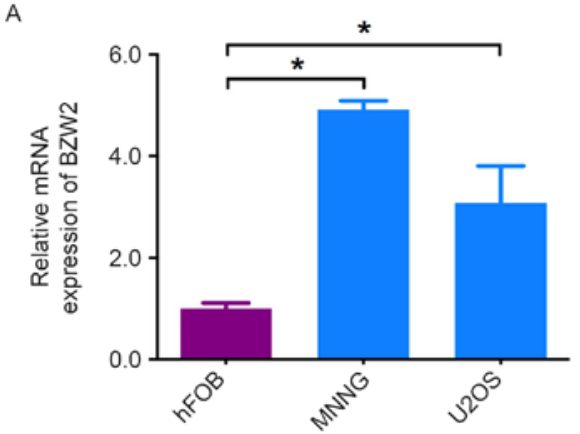

D

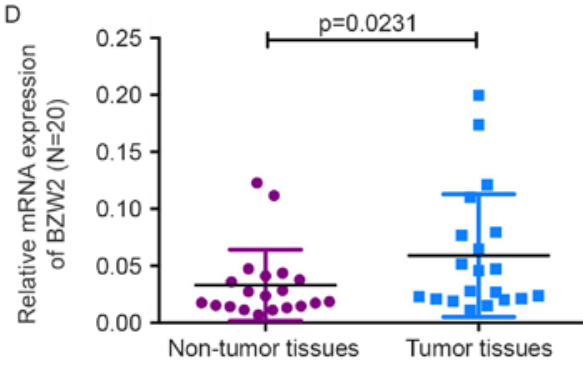

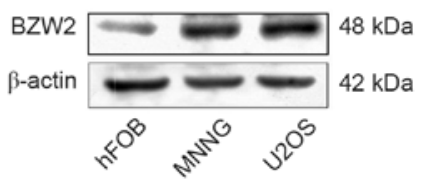

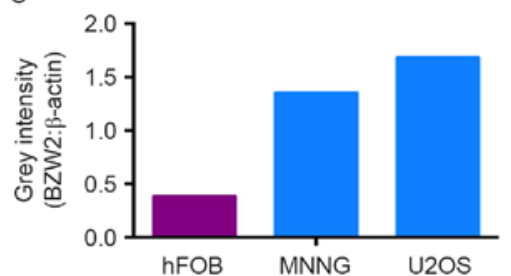

$\mathrm{E}$

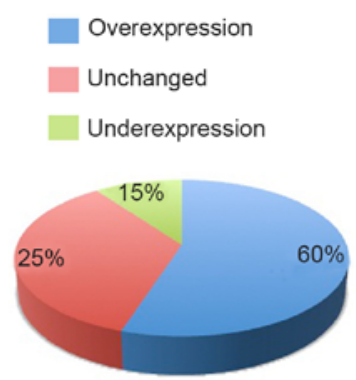

Figure 1. BZW2 was upregulated in osteosarcoma tissues and cell lines. (A and B) The mRNA and protein expression levels of BZW2 were assessed by qRT-PCR and western blotting in osteosarcoma cell lines (MNNG/HOS and U2OS) and a human normal osteoblast cell line (hFOB 1.19). (C) The grey intensity was calculated in the osteosarcoma cell lines and the osteoblast cell line. $\beta$-actin was used as an internal control. (D and E) The expression of BZW2 was detected in 20 pairs of osteosarcoma tissues and their adjacent non-tumor tissues. The expression of BZW2 was overexpressed in osteosarcoma tissues compared with non-tumor tissues.

(Thr308) (1:200; Cell Signaling Technology). Slides were rinsed three times in phosphate-buffered saline (PBS) and incubated for $30 \mathrm{~min}$ using the EnVision Staining kit (DAKO, Glostrup, Denmark), followed by three additional washes in PBS and color development over 3-10 min in a moist chamber at $20^{\circ} \mathrm{C}$ using 3,3'-diaminobenzidine (DAB). The slides were counterstained with hematoxylin and dehydrated in a graded ethyl alcohol series (70,90 and 100\%). For the sections used as negative controls, PBS was used as a substitute for the primary antibody. IHC signal intensities were scored as follows: 0 (no staining), 1 (staining in $<1 \%$ of cells), 2 (staining in $1-10 \%$ of cells), or 3 (staining in $>10 \%$ of cells). The samples classified as 0 and 1 were considered negative, while the samples classified as 2 and 3 were considered positive. Assessment of IHC staining was independently performed by two expert pathologists. Any discordance was resolved by discussion and consensus.

Statistical evaluation. Data were compiled and analyzed using SPSS version 21.0 (SPSS, Inc., Chicago, IL, USA). The differences between groups were evaluated using a two-tailed Student's t-test. The correlations between the IHC results for BZW2 and the clinicopathological parameters were determined using Chi-square tests. The correlation analysis between BZW2 and p-Akt was performed using the Spearman correlation test. $\mathrm{P}<0.05$ was considered significant.

\section{Results}

BZW2 is overexpressed in osteosarcoma cell lines and human osteosarcoma tissue samples. To determine the role of BZW2 in osteosarcoma, we first detected the mRNA and protein expression levels of BZW2 in osteosarcoma cell lines and an osteoblast cell line by qRT-PCR and western blot analyses. Compared with the osteoblast cell line, BZW2 overexpression was detected at both the mRNA and protein levels in osteosarcoma cell lines (Fig. 1A-C). To further confirm its role, BZW2 expression levels were investigated in 20 paired osteosarcoma and adjacent non-tumor tissues. As shown in Fig. 1D and E, BZW2 expression was significantly upregulated in $60 \%(12 / 20)$ of osteosarcoma tissues compared with adjacent non-tumor tissues. These results show that BZW2 may play an important role in osteosarcoma tumorigenesis and thus warrants further investigation.

Knockdown of BZW2 inhibits osteosarcoma cell proliferation in vitro. To explore the functional significance of BZW2 in osteosarcoma, $B Z W 2$-specific siRNA was used to knockdown BZW2 in osteosarcoma cells. BZW2 knockdown was validated by qRT-PCR and western blotting. BZW2 mRNA and protein expression levels were significantly decreased after transfection with BZW2-specific siRNA in MNNG/HOS and U2OS cells (Fig. 2A-D). Next, a CCK-8 assay was used to detect cell proliferation. A 5-day growth curve analysis showed that the knockdown of BZW2 significantly inhibited the growth of osteosarcoma cells (Fig. 2E and F). A colony formation assay was carried out to determine the colony-forming capacity of osteosarcoma cells after the knockdown of BZW2. The number and size of colonies were both obviously decreased in the BZW2-knockdown group in comparison with the control group (Fig. 2G-J). These results demonstrate that BZW2 plays an oncogenic role in osteosarcoma. 

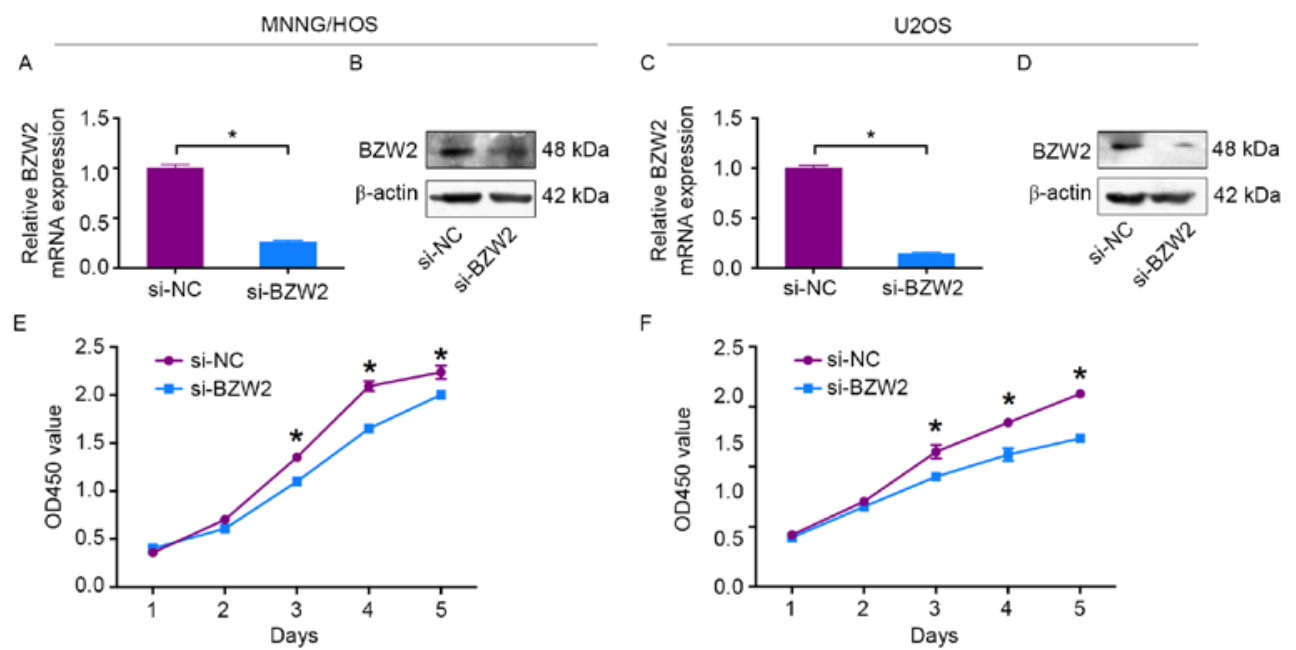

$\mathrm{F}$
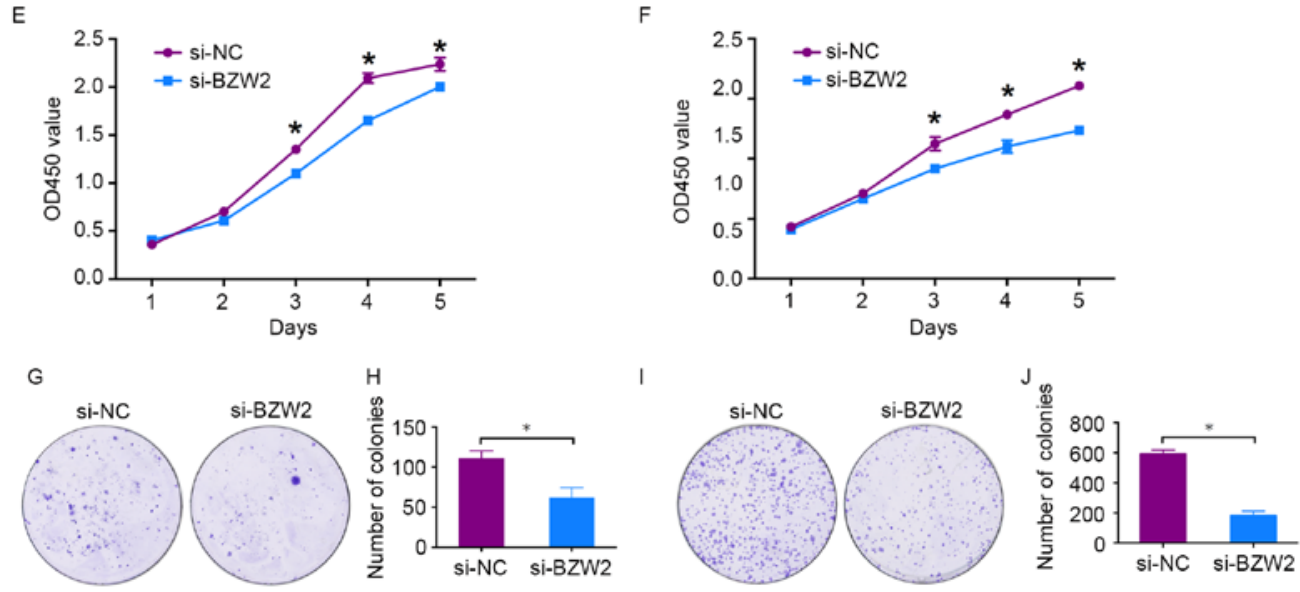

Figure 2. Knockdown of BZW2 inhibits osteosarcoma cell growth in vitro. (A-D) Levels of mRNA and protein expression were validated after si-BZW2 transfection in MNNG/HOS and U2OS cells by qRT-PCR and western blotting, respectively. (E and F) Cell Counting Kit-8 (CCK-8) assays were performed after siRNA transfection. (G-J) Colony formation assays were performed using BZW2-silenced osteosarcoma cells and control cells. Data are representative of results from three independent experiments' ${ }^{\prime} \mathrm{P}<0.05$.
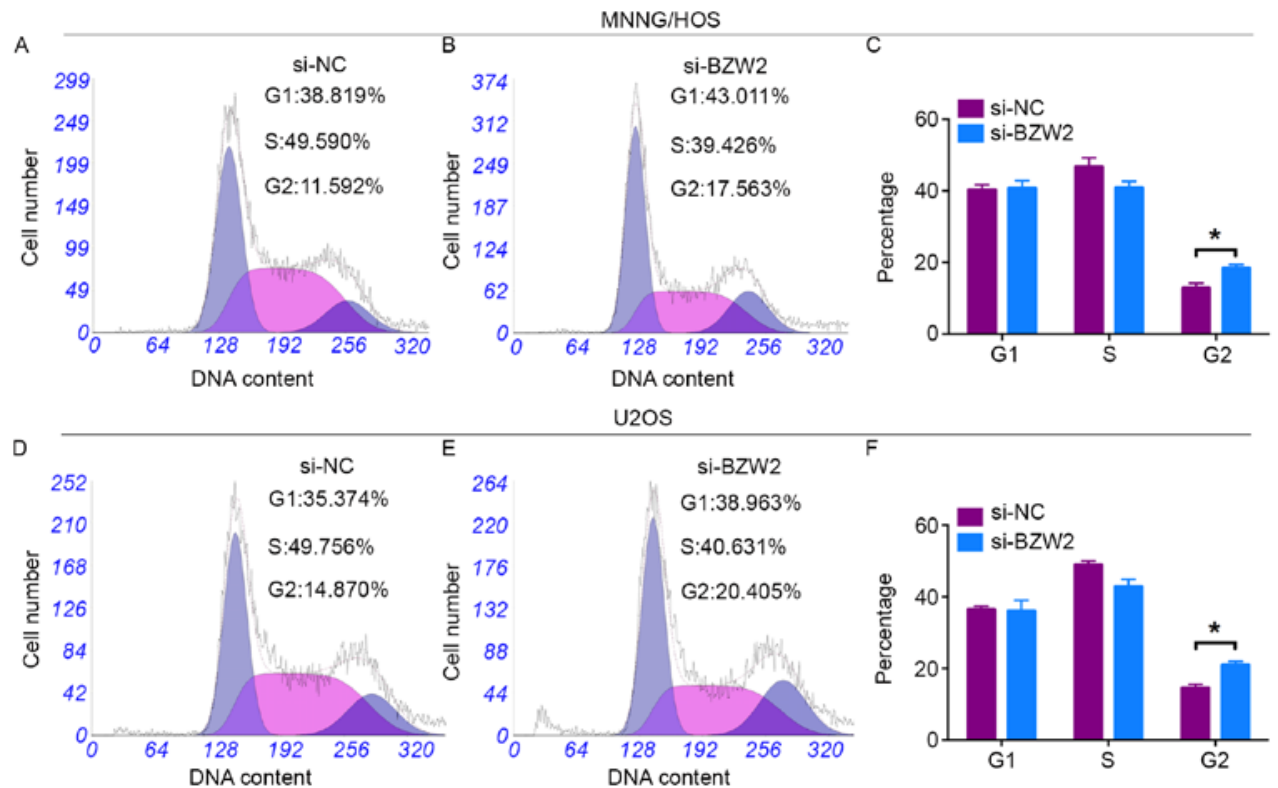

Figure 3. Knockdown of BZW2 inhibits G2/M cell cycle transition in osteosarcoma cells. Representative images of the cell cycle assay results for MNNG/HOS cells (A and B), and U2OS cells (D and E) after transfection with si-NC or si-BZW2. (C and F) Diagrams showing the results of the cell cycle assays for MNNG/HOS and U2OS cells; ${ }^{*} \mathrm{P}<0.05$.

Knockdown of BZW2 inhibits the G2/M cell cycle transition in osteosarcoma cells. Cell cycle changes in response to BZW2 knockdown were analyzed using flow cytometry. A cell cycle assay revealed that si-BZW2-transfected cells had a significant higher percentage of cells in the G2/M phase than that of si-NC-transfected cells (Fig. 3A-F). However, no significant difference was found in the $\mathrm{G} 1$ or $\mathrm{S}$ cell populations between BZW2-knockdown and control-transfected cells.
In conclusion, these data revealed that BZW2 knockdown attenuated osteosarcoma cell proliferation by inhibiting the G2/M cell cycle transition.

Knockdown of BZW2 inhibits osteosarcoma cell proliferation via the AKT/mTOR signaling pathway. To determine the mechanism by which BZW2 affects the proliferation of osteosarcoma cells, intracellular signaling arrays were used to detect 
A

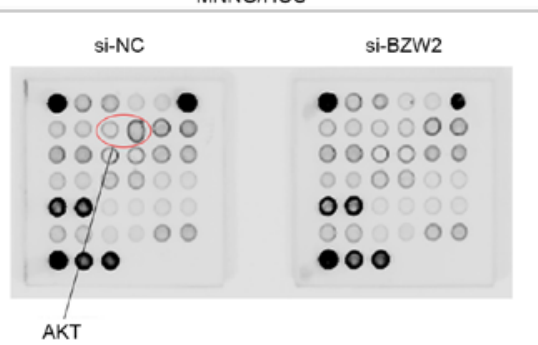

B

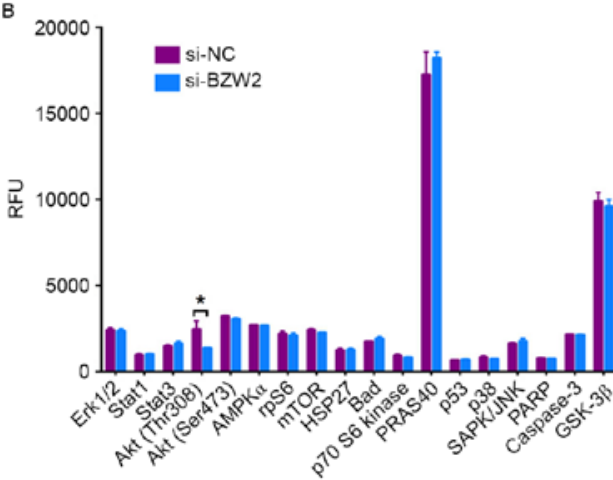

C

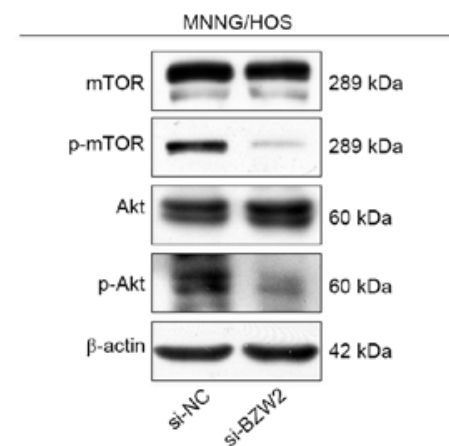

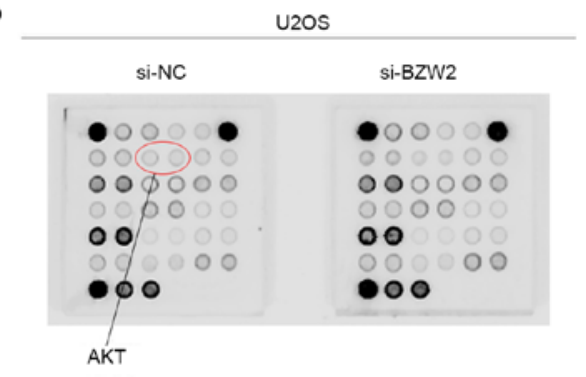

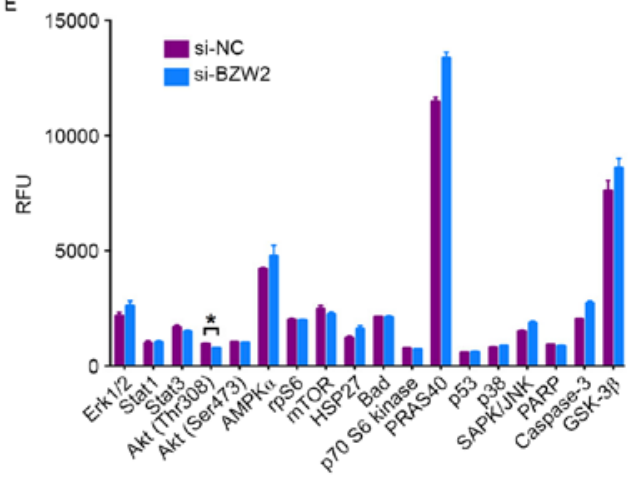

$\mathrm{F}$

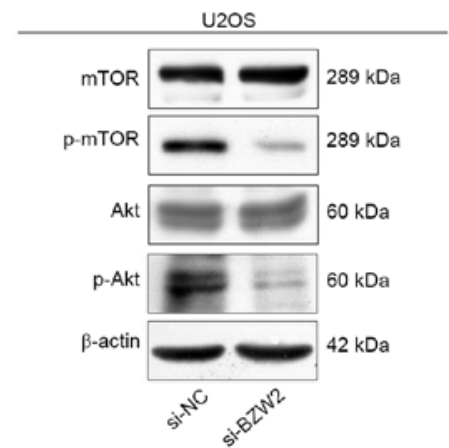

Figure 4. Knockdown of BZW2 inhibits the Akt/mTOR signaling pathway in osteosarcoma cells. (A and D) MNNG/HOS and U2OS cell extracts were prepared and analyzed using the PathScan Intracellular Signaling Antibody Array kit (\#7744). Images were captured using the Odyssey ${ }^{\circledR}$ Infrared Imaging System (LI-COR). (B and E) Diagrams show the quantitative results for MNNG/HOS and U2OS cells. (C and F) Western blot analysis of the Akt/mTOR signaling pathway, including mTOR and Akt, in MNNG/HOS and U2OS cells transfected with si-NC or si-BZW2.

A
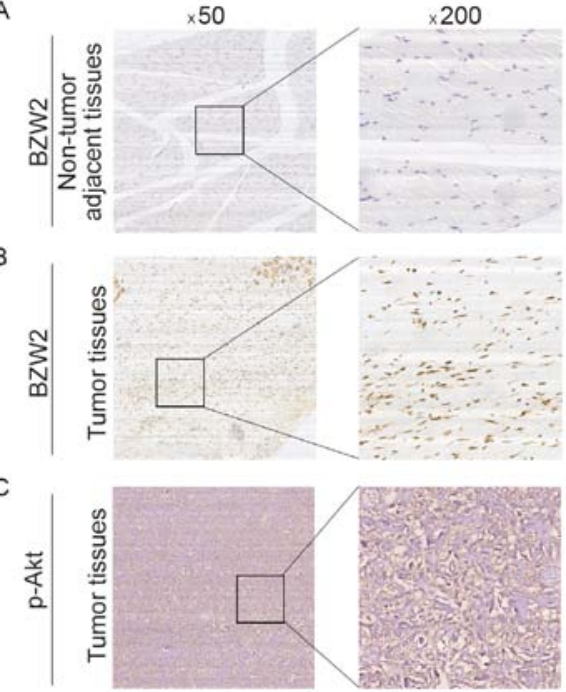

Figure 5. Clinical significance of BZW2 in patients with osteosarcoma (A) Representative IHC image of BZW2 expression in non-tumor adjacent tissues. (B) Representative IHC image of BZW2 expression in osteosarcoma tissues. (C) Representative IHC image of p-Akt expression in osteosarcoma tissues. Original magnification, x50 and x200. protein expression after BZW2 knockdown in MNNG/HOS and U2OS cells. A small decrease in the phosphorylation of Akt-Thr308 was detected in osteosarcoma cells (Fig. 4A-D). We then examined whether BZW2 influences the AKT/mTOR signaling pathway in osteosarcoma cells using western blot analysis. Based on this analysis, BZW2 knockdown inhibited the phosphorylation of Akt and mTOR. These results support the conclusion that BZW2 plays a crucial role in osteosarcoma cell growth by influencing the Akt/mTOR signaling pathway.

BZW2 expression is correlated to Enneking stage and tumor recurrence in osteosarcoma. To further determine the clinicopathological significance of BZW2 in osteosarcoma, we performed an IHC analysis of BZW2 using 50 human osteosarcoma tissue samples and corresponding non-tumor tissues. Representative IHC images of BZW2 expression in osteosarcoma tissues and adjacent non-tumor tissues are shown in Fig. 5. Correlations between the BZW2 expression level and the clinicopathological characteristics of patients with osteosarcoma are summarized in Table I. BZW2 expression levels were higher in osteosarcoma tissue samples than in 
Table I. Correlation analyses of BZW2 protein expression in relation to clinicopathological variables of 50 patients with osteosarcoma.

\begin{tabular}{|c|c|c|c|c|c|}
\hline \multirow{2}{*}{$\begin{array}{l}\text { Clinicopatho- } \\
\text { logical } \\
\text { parameters }\end{array}$} & \multirow{2}{*}{$\begin{array}{l}\text { No. of } \\
\text { cases }\end{array}$} & \multicolumn{2}{|c|}{$\begin{array}{c}\text { BZW2 expression } \\
\text { level }\end{array}$} & \multirow[b]{2}{*}{$\chi^{2}$} & \multirow[b]{2}{*}{ P-value } \\
\hline & & Negative & Positive & & \\
\hline Sex & & & & 1.602 & 0.206 \\
\hline Male & 29 & 19 & 10 & & \\
\hline Female & 21 & 10 & 11 & & \\
\hline Age (years) & & & & 0.512 & 0.474 \\
\hline$<18$ & 28 & 15 & 13 & & \\
\hline$\geq 18$ & 22 & 14 & 8 & & \\
\hline Location & & & & 1.667 & 0.434 \\
\hline Femur & 24 & 12 & 12 & & \\
\hline Tibia & 14 & 10 & 4 & & \\
\hline Elsewhere & 12 & 7 & 5 & & \\
\hline $\begin{array}{l}\text { Tumor necrosis } \\
\text { rate }(\%)\end{array}$ & & & & 0.006 & 0.939 \\
\hline$<90$ & 36 & 21 & 15 & & \\
\hline$\geq 90$ & 14 & 8 & 6 & & \\
\hline $\begin{array}{l}\text { Cortical } \\
\text { destruction }\end{array}$ & & & & 0.487 & 0.485 \\
\hline Yes & 38 & 21 & 17 & & \\
\hline No & 12 & 8 & 4 & & \\
\hline Recurrence & & & & 6.226 & $0.013^{\mathrm{a}}$ \\
\hline Yes & 23 & 9 & 14 & & \\
\hline No & 27 & 20 & 7 & & \\
\hline Metastasis & & & & 0.112 & 0.738 \\
\hline Yes & 32 & 18 & 14 & & \\
\hline No & 18 & 11 & 7 & & \\
\hline $\begin{array}{l}\text { Ennecking } \\
\text { stage }\end{array}$ & & & & 6.912 & $0.009^{\mathrm{a}}$ \\
\hline II & 34 & 24 & 10 & & \\
\hline III & 16 & 5 & 11 & & \\
\hline
\end{tabular}

${ }^{\mathrm{a}} \mathrm{P}<0.05$. BZW2, basic leucine zipper and W2 domains 2.

corresponding non-tumor tissues $(\mathrm{P}=0.021)$. The expression level of BZW2 was higher in patients at a clinically advanced Enneking stage than in those at an early stage $(\mathrm{P}=0.009)$. The BZW2 expression level was also positively correlated with recurrence $(\mathrm{P}=0.013)$. No correlation was found between BZW2 and other demographic and clinical factors, including sex, age, tumor location, tumor necrosis rate, cortical destruction and metastasis. Finally, we examined p-Akt expression in osteosarcoma tissues. Representative images of p-Akt expression in osteosarcoma tissues are shown in Fig. 5C. We found that there was a significant correlation between the expression of both BZW2 and p-Akt in osteosarcoma tissues $(\mathrm{R}=0.380$, $\mathrm{P}=0.006)$. Collectively, these results indicate that BZW2 is upregulated in osteosarcoma and plays a potentially important role in osteosarcoma progression.

\section{Discussion}

Osteosarcoma exhibits a propensity to affect children and adolescents. The treatment for osteosarcoma has advanced from amputation to complex limb salvage surgery combined with systemic multiagent chemotherapy (12). However, robust evidence indicates that in the 30 years since the dramatic increase in survival in the late 1980s, the 5-year survival rate for osteosarcoma has not improved substantially (13). Thus, it is important to identify additional potential therapeutic targets. ROCK1 has been reported as a potential therapeutic target in osteosarcoma. The knockdown of ROCK1 inhibits proliferation and induces apoptosis in osteosarcoma cell lines (14). GLI2 regulates metastasis as well as progression in osteosarcoma. The depletion of GLI2 may be an effective therapeutic method for preventing osteosarcoma metastasis (15). In addition, ROR2 and Trps1 are potential therapeutic targets $(16,17)$.

In the present study, we demonstrated that BZW2 was upregulated in osteosarcoma cell lines and osteosarcoma tissue samples in comparison with corresponding adjacent non-tumor tissues. The knockdown of BZW2 impaired osteosarcoma cell proliferation as well as colony-formation ability, and led to cell cycle arrest in the $\mathrm{G} 2 / \mathrm{M}$ phase. These results indicated that $B Z W 2$ plays a critical role in osteosarcoma cell growth and functions as an oncogene in osteosarcoma. Notably, in the present study, we found that the expression of BZW2 is positively correlated with osteosarcoma recurrence and Enneking stage according to IHC staining results. BZW2 plays a role in the regulation of translation initiation. Singh et al reported that BZW2, also termed 5MP1, interacts with human translation factor eIF2 and eIF3 in vitro and promotes ternary complex formation (18). Subsequent research revealed that the expression of BZW2 could induce ATF4 translation, which plays an important role in eukaryotic gene regulation (19). ATF4 is a pro-oncogenic transcription factor that drives the transcription of various genes involved in amino acid synthesis, nutrient uptake, autophagy and the inhibition of apoptosis (20).

According to a combined analysis of murine and human gene expression using microarray and chip analyses, BZW2 is associated with the ability of MYC to maintain tumorigenesis (21). However, the potential mechanism by which BZW2 functions in osteosarcoma remains far from clear. In the present study, to further investigate the underlying mechanisms of BZW2, we used the PathScan Intracellular Signaling Antibody Array kit to identify the host signaling pathway affected by BZW2 depletion. These data revealed a small decrease in the phosphorylation of some components of the Akt/mTOR pathway in BZW2 knockdown cells. The Akt/mTOR signaling pathway is tightly associated with progression in various types of cancer, such as breast (8), lung (22) and prostate cancer (23). Recently, the Akt/mTOR signaling pathway was also found to participate in the tumorigenesis of osteosarcoma. Song et al reported that p53 suppresses osteosarcoma cell growth, metastasis and angiogenesis via the inhibition of the PI3K/AKT/mTOR signaling pathway (24). Upon phosphorylation, activated mTOR contributes to osteosarcoma cellular transformation and a poor prognosis (25). We detected changes in the Akt/mTOR pathway after BZW2 knockdown by western blotting. Specifically, the knockdown of BZW2 inhibited the expression of phosphorylated Akt and mTOR, indicating that BZW2 
influences osteosarcoma progression partly via the Akt/mTOR signaling pathway. The possible mechanism of BZW2 interaction with Akt/mTOR signaling pathway is as follows. It was reported that BZW2 induced ATF4 translation (19). ATF4 and phosphorylated Akt are important endoplasmic reticulum (ER) stress proteins (26). Therefore, BZW2 may interact with ATF4 to regulate the Akt/mTOR signaling pathway.

In conclusion, we demonstrated for the first time that BZW2 is upregulated in human osteosarcoma cell lines and clinical tissues. High expression of BZW2 was positively correlated to the Enneking stage and the recurrence of osteosarcoma, and these correlations may be explained by its effects on cell proliferation. More importantly, we demonstrated that BZW2 has an oncogenic function by affecting the Akt/mTOR signaling pathway in osteosarcoma cells. These data provide compelling evidence that BZW2 is a potential therapeutic target for osteosarcoma.

\section{Acknowledgements}

The manuscript was edited by Elsevier English language editing service.

\section{References}

1. Morrow JJ and Khanna C: Osteosarcoma genetics and epigenetics: Emerging biology and candidate therapies. Crit Rev Oncog 20: 173-197, 2015.

2. Klein MJ and Siegal GP: Osteosarcoma: Anatomic and histologic variants. Am J Clin Pathol 125: 555-581, 2006.

3. Yamamoto $\mathrm{N}$ and Tsuchiya $\mathrm{H}$ : Chemotherapy for osteosarcoma - where does it come from? What is it? Where is it going? Expert Opin Pharmacother 14: 2183-2193, 2013.

4. Ferrari S and Serra M: An update on chemotherapy for osteosarcoma. Expert Opin Pharmacother 16: 2727-2736, 2015.

5. Geller DS and Gorlick R: Osteosarcoma: A review of diagnosis, management, and treatment strategies. Clin Adv Hematol Oncol 8: 705-718, 2010

6. Guo Z, Neilson LJ, Zhong H, Murray PS, Zanivan S and Zaidel-Bar R: E-cadherin interactome complexity and robustness resolved by quantitative proteomics. Sci Signal 7: rs7, 2014.

7. Li S, Chai Z, Li Y, Liu D, Bai Z, Li Y, Li Y and Situ Z: BZW1, a novel proliferation regulator that promotes growth of salivary muocepodermoid carcinoma. Cancer Lett 284: 86-94, 2009.

8. Polo ml, Riggio M, May M, Rodríguez MJ, Perrone MC, Stallings-Mann M, Kaen D, Frost M, Goetz M, Boughey J, et al: Activation of PI3K/Akt/mTOR signaling in the tumor stroma drives endocrine therapy-dependent breast tumor regression. Oncotarget 6: 22081-22097, 2015.

9. Sharma N, Nanta R, Sharma J, Gunewardena S, Singh KP, Shankar S and Srivastava RK: PI3K/AKT/mTOR and sonic hedgehog pathways cooperate together to inhibit human pancreatic cancer stem cell characteristics and tumor growth. Oncotarget 6: 32039-32060, 2015.

10. Sun CH, Chang YH and Pan CC: Activation of the PI3K/Akt/ mTOR pathway correlates with tumour progression and reduced survival in patients with urothelial carcinoma of the urinary bladder. Histopathology 58: 1054-1063, 2011.
11. Costa C, Pereira S, Lima L, Peixoto A, Fernandes E, Neves D, Neves M, Gaiteiro C, Tavares A, Gil da Costa RM, et al: Abnormal protein glycosylation and activated PI3K/Akt/mTOR pathway: Role in bladder cancer prognosis and targeted therapeutics. PLoS One 10: e0141253, 2015.

12. Isakoff MS, Bielack SS, Meltzer P and Gorlick R: Osteosarcoma: Current treatment and a collaborative pathway to success. J Clin Oncol 33: 3029-3035, 2015.

13. Whelan J, McTiernan A, Cooper N, Wong YK, Francis M, Vernon S and Strauss SJ: Incidence and survival of malignant bone sarcomas in England 1979-2007. Int J Cancer 131: E508-E517, 2012.

14. Liu X, Choy E, Hornicek FJ, Yang S, Yang C, Harmon D, Mankin $\mathrm{H}$ and Duan Z: ROCK1 as a potential therapeutic target in osteosarcoma. J Orthop Res 29: 1259-1266, 2011.

15. Nagao-Kitamoto H, Nagata M, Nagano S, Kitamoto S, Ishidou $Y$, Yamamoto T, Nakamura S, Tsuru A, Abematsu M, Fujimoto Y, et al: GLI2 is a novel therapeutic target for metastasis of osteosarcoma. Int J Cancer 136: 1276-1284, 2015.

16. Morioka K, Tanikawa C, Ochi K, Daigo Y, Katagiri T, Kawano H, Kawaguchi H, Myoui A, Yoshikawa H, Naka N, et al: Orphan receptor tyrosine kinase ROR2 as a potential therapeutic target for osteosarcoma. Cancer Sci 100: 1227-1233, 2009.

17. Li Z, Jia M, Wu X, Cui J, Pan A and Li L: Overexpression of Trps1 contributes to tumor angiogenesis and poor prognosis of human osteosarcoma. Diagn Pathol 10: 167, 2015.

18. Singh CR, Watanabe R, Zhou D, Jennings MD, Fukao A, Lee B, Ikeda Y, Chiorini JA, Campbell SG, Ashe MP, et al: Mechanisms of translational regulation by a human eIF5-mimic protein. Nucleic Acids Res 39: 8314-8328, 2011.

19. Hiraishi H, Oatman J, Haller SL, Blunk L, McGivern B, Morris J, Papadopoulos E, Gutierrez W, Gordon M, Bokhari W, et al: Essential role of eIF5-mimic protein in animal development is linked to control of ATF4 expression. Nucleic Acids Res 42: 10321-10330, 2014.

20. Harding HP, Zhang Y, Zeng H, Novoa I, Lu PD, Calfon M, Sadri N, Yun C, Popko B, Paules R, et al: An integrated stress response regulates amino acid metabolism and resistance to oxidative stress. Mol Cell 11: 619-633, 2003.

21. Wu CH, Sahoo D, Arvanitis C, Bradon N, Dill DL and Felsher DW: Combined analysis of murine and human microarrays and ChIP analysis reveals genes associated with the ability of MYC to maintain tumorigenesis. PLoS Genet 4: e1000090, 2008.

22. Yu G, Huang B, Chen G and Mi Y: Phosphatidylethanolaminebinding protein 4 promotes lung cancer cells proliferation and invasion via PI3K/Akt/mTOR axis. J Thorac Dis 7: 1806-1816, 2015.

23. Xia Q, Zheng Y, Jiang W, Huang Z, Wang M, Rodriguez R and Jin X: Valproic acid induces autophagy by suppressing the Akt/mTOR pathway in human prostate cancer cells. Oncol Lett 12: 1826-1832, 2016.

24. Song R, Tian K, Wang W and Wang L: P53 suppresses cell proliferation, metastasis, and angiogenesis of osteosarcoma through inhibition of the PI3K/AKT/mTOR pathway. Int J Surg 20: 80-87, 2015.

25. Hu K, Dai HB and Qiu ZL: mTOR signaling in osteosarcoma: Oncogenesis and therapeutic aspects (Review). Oncol Rep 36: 1219-1225, 2016.

26. Hasanain M, Bhattacharjee A, Pandey P, Ashraf R, Singh N, Sharma S, Vishwakarma AL, Datta D, Mitra K and Sarkar J: $\alpha$-Solanine induces ROS-mediated autophagy through activation of endoplasmic reticulum stress and inhibition of $\mathrm{Akt} / \mathrm{mTOR}$ pathway. Cell Death Dis 6: e1860, 2015. 